\title{
Investigation of Catheter Curvature and Genetic Algorithms in Conductance Catheter Optimization
}

\author{
C. Thaijiam, and T. J. Gale
}

\begin{abstract}
Catheter curvature affects accuracy of intraventricular blood volume measurement when using conductance catheter techniques, especially with irregular geometries, such as in the right ventricle. To investigate this effect, we present results from using different curved catheter configurations and different numbers of electrodes in a simple Finite Element model. It was found that there is an apparent increase in accuracy with curvature, due to greater linearity in the field in the region of the measurement electrodes, which are located farther from the source electrodes as curvature increases. Also, optimization using Genetic Algorithms is presented as a method to find the optimal distribution of measurement electrodes. We plan to extend these results to develop improved electrode configurations for using in blood volume measurement in the right ventricle.
\end{abstract}

\section{INTRODUCTION}

$\mathrm{W}$ E have previously used analytical and numerical techniques to investigate the electric potential field distribution in a uniform conductor with both single and dual excitation at asymmetrical positions $[1,2]$. Results show that the electric field distribution from using point current excitation is non-uniform. This can cause reduced accuracy of intra-ventricular blood volume measurement using conductance catheter techniques. We are interested in using conductance catheters to measure intra-ventricular blood volume in the right ventricle, and this may necessitate use of a curved catheter to span the whole chamber, due to the crescent shape of the right ventricle [3]. Investigation of the effect of conductance catheter location on volume measurement has been exposed in our previous work [4]. Nevertheless, investigating curved catheter configurations and using optimization methods has not yet been reported.

Here we present results from using different curved catheter configurations and different numbers of electrodes in a simple Finite Element model. Also, optimization using Genetic Algorithms is presented as a method to find the optimal distribution of measurement electrodes. Regular geometry is used in the model so that principles can be investigated without the potentially confounding effects of irregular geometry.

Manuscript received March 30, 2007.

C. Thaijiam is with School of Engineering, University of Tasmania, Hobart, Tasmania, Australia, on leave from Faculty of Engineering, Srinakharinwirot University, Nakhornnayok, Thailand (phone: +61-3-62262142; fax: +61-3-6226-7247; e-mail: ct0@utas.edu.au).

T.J. Gale is with School of Engineering, University of Tasmania, Hobart, Tasmania, Australia. (e-mail: T.Gale@utas.edu.au).

\section{Methodology}

\section{A. Model}

The model comprised a conductor of square cross section, dimension $2 L \times D \times D$ (Fig. 1). The conductance catheter was represented by exciting electrodes (source and sink) positioned at asymmetrical positions, plus a variable number of measurement electrodes distributed along a curve between the source and sink.

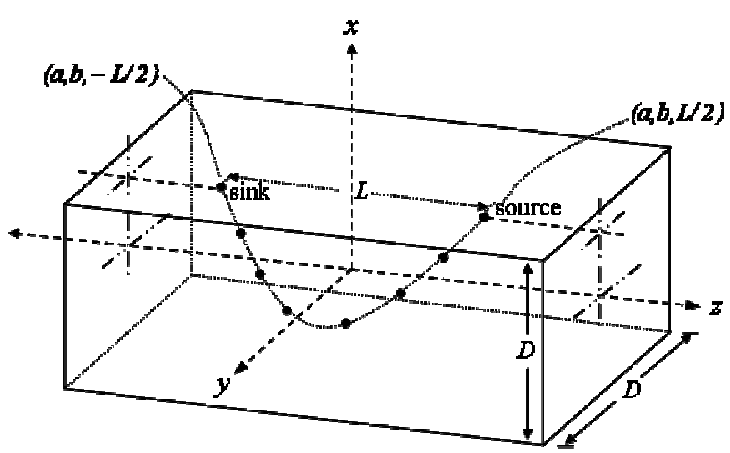

Fig. 1: Sink and source electrodes in the finite element model

\section{B. Conductance Technique Theory}

The conductance catheter divided the ventricle into segments as is shown in Fig. 2. Constant electric current was injected through the source and sink electrodes, and the segmental resistance was calculated knowing the potential drop across each volume segment [5] as follows:

$$
R_{i}=\frac{\rho L_{i}}{A_{i}}=\frac{\rho L_{i}{ }^{2}}{A_{i} L_{i}}=\frac{\rho L_{i}{ }^{2}}{V o l_{i}},
$$

where $\quad R_{i}=$ segmental resistance, $\rho=$ resistivity, $L_{i}=$ effective spacing between electrodes, $A_{i}=$ cross sectional area of the segment and $\mathrm{Vol}_{i}=$ segmental volume.

Effective spacing was defined as the distance between adjacent measurement electrodes in the $\vec{a}_{N}$ direction (Fig. 3),

$$
\text { Effective spacing }(i)=\left(\vec{L} c_{i}-\vec{L} c_{i-1}\right) \cdot \vec{a}_{N}
$$




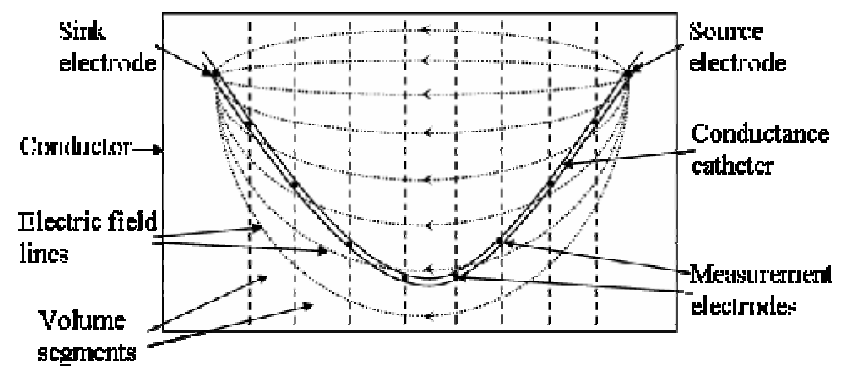

Fig. 2: Model of a conductance catheter inserted into the model

where Effective spacing $(i)=$ segmental effective spacings, and $\vec{L} c_{i}=$ the vectors between adjacent measurement electrodes directed along the curved catheter as shown in Fig. 3. Use of effective spacing improved the accuracy of the volume calculation [4].

An expression for segmental volume may be found from (1),

$$
\operatorname{Vol}_{i}=\frac{\rho L_{i}^{2}}{R_{i}}
$$

Knowing the segment volumes, we can find the total ventricular volume, $\mathrm{Vol}_{\text {total }}$,

$$
V o l_{\text {total }}=\sum_{i=1}^{p} V_{\text {ol }},
$$

where $p=$ total number of volume segments.

\section{Finite Element Solution to the Potential Field}

The electric potential field distribution inside the model was found using Poisson's equation [5],

$$
\nabla^{2} V=-I / \sigma
$$

where $V=$ electric potential, $\sigma=$ conductivity and $I=$ current source density.

The problem was configured with $L=D=50 \mathrm{~mm}$. A current source $\left(I_{0}\right)$ of $20 \mu \mathrm{A}$ was applied on the source electrode. The current sink was constrained by a voltage of zero, and conductor conductivity was $0.67 \mathrm{~S} / \mathrm{m}$ [6]. The current source and current sink were at $x=a=8 D / 20, y$ $=b=0$, and $z= \pm L / 2$, respectively.

The Finite Element method was used to find numerical solutions for the model, utilizing ANSYS Multiphysics version 10 software (ANSYS, Inc., Canonsburg, USA). The conductor geometry was represented by 132,496 tetrahedral solid elements, and (5) was solved for source and sink electrodes represented by individual nodes. The resulting electric potential values were substituted into (3). (4) was then used to find the segmental volumes and the total volume within the region of the conductor spanned by the measurement electrodes.

\section{Conductance Catheter Configurations}

Three positions, current source, current sink, and Extreme position were used to configure the curved catheter (Fig. 3). The Extreme position was the point on the catheter that was the furthest distance from the axis $\left(\vec{a}_{N}\right)$ joining the current sink and source positions, and was constrained to lie on the $x$-axis. Cubic spline interpolation was used to define the curve of the catheter from these three points [7]. The first and last measurement electrodes were located on the z-axis at $L / 2-0.005$ and $-L / 2+0.005$, respectively. Also, the number of measurement electrodes used $(2,4,6,8$, and 10) was varied to investigate the effect on volume error.

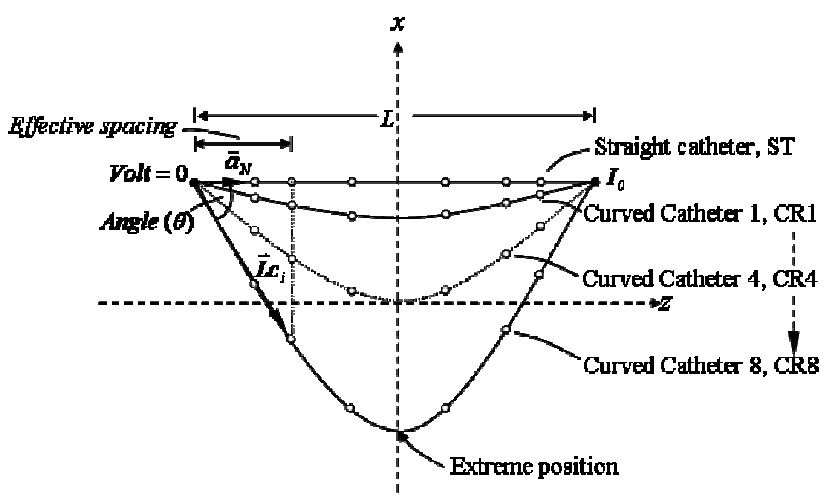

Fig. 3: Configurations of conductance catheter curvature (the example shows 6 measurement electrodes)

\section{E. Genetic Algorithm to Optimize Catheter Design}

Genetic Algorithms [8] were used to solve for optimal effective spacings, using MATLAB and the Genetic Algorithm Toolbox (Mathworks, Massachusetts, USA). Catheter configurations were optimized by varying the position of the measurement electrodes along the catheter. Both the source and sink positions remained fixed, while the extreme position varied from $x=+0.02$ (straight) to $x=$ $0.02 \mathrm{~mm}$ (maximum curvature).

The percent true volume error $\left(\varepsilon_{t}\right)$ was used as the fitness value function for the standard Genetic Algorithm method. The optimization method involved calculation of this value for different locations of "randomly" chosen measurement electrodes, with the optimum location being the first configuration satisfying the fitness value function.

A summary of the optimization method follows, with further details in Fig. 4. First, discrete data such as node numbers, element numbers, and nodal potential values were transferred from the Finite Element model. Then, for each calculation, the procedure involved the following steps. Measurement electrode locations along the catheter were chosen. Solid angle theory [9] was then used to identify which elements from the Finite Element model enclosed the catheter measurement electrodes. Quadratic interpolation 


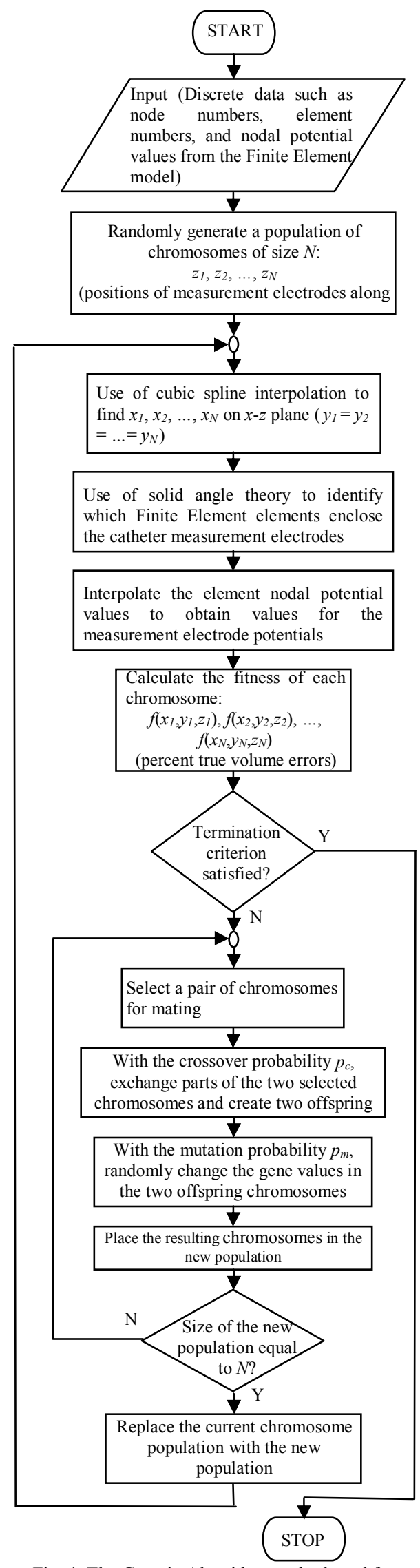

Fig. 4: The Genetic Algorithm method used for optimizing percent true volume error using conductance catheter techniques was then used to linearly interpolate the element nodal potential values to obtain values for the measurement electrode potentials. Conductance volumes were then determined for different measurement electrode configurations, and $\varepsilon_{t}$ calculated by comparing with the known exact volume of the conductor. If this value satisfied the fitness value function, then processing was stopped, otherwise, the procedure was repeated.

\section{RESULTS}

Results of investigations varying the Extreme position and the number of measurement electrodes are summarized in Fig. 5. There is a progressive decrease in volume error with catheter curvature and also as the number of electrodes is increased.

The electric potential field distribution between the first and last measurement electrode of each catheter is shown in Fig. 6. This shows the field is non-linear near the source and sink and relative linear elsewhere, and also how the range of the potential values decreased with catheter curvature.

Fig. 7 shows optimal distributions of measurement electrodes on catheters with different values of extreme

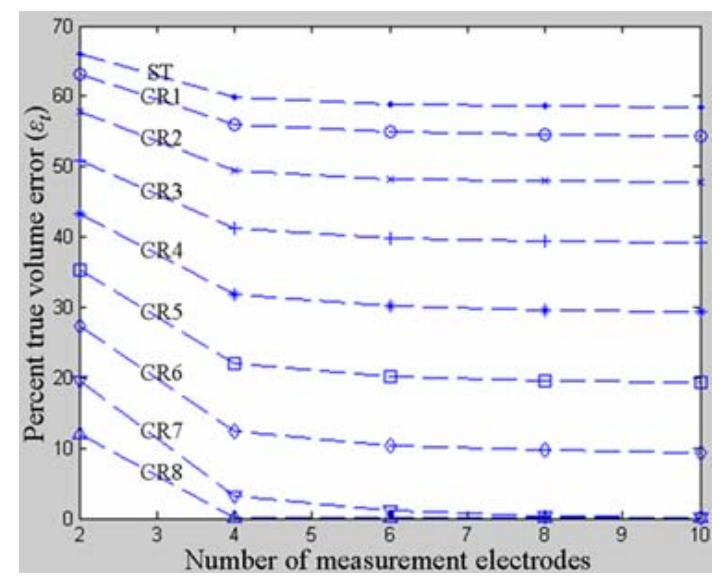

Fig. 5: Percent true volume errors versus number of measurement electrodes

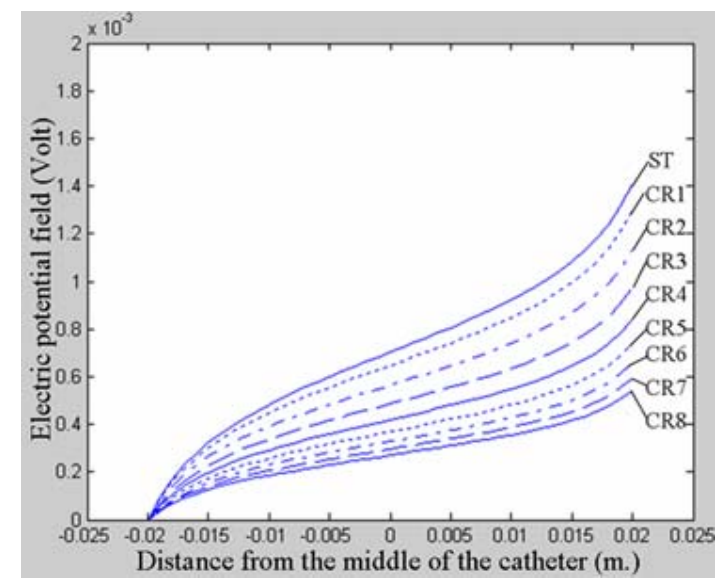

Fig. 6: The electric potential field distribution between the first and last measurement electrode for catheters of different curvature 


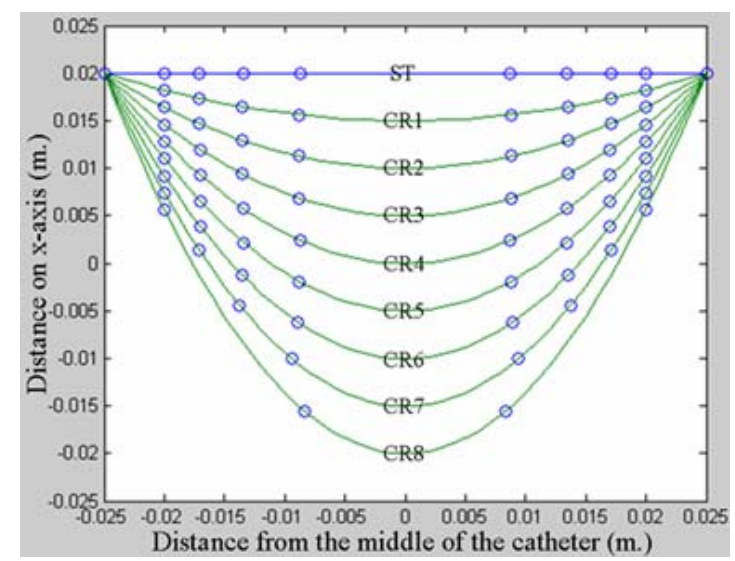

Fig. 7: Optimal measurement electrode distributions position. The location of the eight measurement electrodes is clearly shown for configurations ST and CR1 - CR7. However, for CR8, optimal measurement was found using only four discrete positions (with three electrodes located at each of the two positions adjacent to the Extreme position).

\section{DISCUSSION}

Results from Fig 5 show that volume error decreases as the Extreme point is displaced from the straight catheter location. i.e. Volume error reduces with catheter curvature.

Investigations varying the number of measurement electrodes (Fig. 5) show that when the number of measurement electrodes increases, volume error decreases. However, using more than eight measurement electrodes results in little improvement in volume error. Also, for curved catheter configuration 8 (CR8), after the number of measurement electrodes is equal to 4 , percent true volume error reaches a constant.

The decrease in volume error with both increased curvature and number of electrodes may be explained by considering the (well-known) behavior of the electric field, where the electric potential field distribution is non-uniform but becomes more uniform with distance from the exciting electrodes $[1,2]$. The magnitude of the electric potential gradient is very high near exciting electrodes (source and sink) and decreases gradually until it is relatively constant near the middle of the conductor. This effect can be seen in the potential field distribution along each catheter (Fig. 6).

Also, using equally spaced measurement electrodes is not ideal for achieving the most accurate volume measurement. Fig. 7 shows that optimization of electrode positions results in higher electrode densities nearer the source and sink electrodes (the regions where the potential field is most nonlinear - refer Fig. 6).

The apparent increase in accuracy with curvature is therefore most likely due simply to the greater linearity of the field in the region of the measurement electrodes.

Similarly, for a particular curvature, the increase in accuracy achieved by an unequal distribution of electrodes over the length of the catheter, is likely to be due to the better representation of the field achieved by having more closely spaced electrodes in the regions of greatest nonlinearity.

Use of curved catheter configurations resulted in more accurate measurement of conductance volume than use of a standard straight configuration for the model used in this study. However, whether this applies to other geometries may depend on achieving optimal positions of measurement electrodes for the particular field distributions found in each particular case, and will be the subject of future research.

Validation against a realistic heart model and also against experimental results are warranted, especially considering the simple model presented here represents a cuboidal shaped isolated ventricle without surrounding structures. Also, the optimization results obtained here using Genetic Algorithms should be compared to further results obtained applying traditional optimization algorithms.

\section{CONCLUSION}

There is scope for use of the Finite Element method and Genetic Algorithms to improve the accuracy of conductance catheter volume measurements by strategic placement of measurement electrodes. We hope to use knowledge from this investigation to optimize conductance curvature for the purpose of achieving more accurate measures of blood volume within the right ventricle. Future work will involve applying a numerical model to realistically shaped geometries of the right ventricle, and also combining curvature optimization with the existing Genetic Algorithm based electrode distribution optimization methods.

\section{REFERENCES}

[1] C. Thaijiam, and T. J. Gale, "Analytical and numerical studies of volume measurement using conductance catheter techniques," in Proceedings of the 2005 IEEE, Engineering in Medicine and Biology 27th Annual Conference, Shanghai, China, pp. 2291-2294, 2005.

[2] C. Thaijiam, and T. J. Gale, "Studies of Single and Dual Excitation using Conductance Catheter Techniques," in Proceedings of the 2006 IASTED, The Fourth International Conference on Biomechanical Engineering, Innsbruck, Austria, pp. 294-299, 2006.

[3] B. Gopakumaran, J. H. Peter, S. Krucinski, and P. A. Murray, "Realtime continuous measurement of right ventricular volume using a conductance catheter," Biomedical instrumentation \& Technology, vol. 30, no. 5, pp. 427-438, September/October 1996.

[4] C. Thaijiam, and T. J. Gale, "The Effect of Conductance Catheter Curvature on Volume Measurement," in Proceedings of the 2006 ISBME, The second International Symposium on Biomedical Engineering, Bangkok, Thailand, pp. 168-170, 2006.

[5] W. H. Hayt, JR., Engineering electromagnetics, Fifth edition, McGraw-Hill, Inc., 1989.

[6] L. A. Geddes, and L.E. Baker, "The specific resistance of biological material: a compendium of data for the biomedical engineer and physiologist," Med. Biol. Eng., vol.5, pp.271-293, 1967.

[7] S. C. Chapra, and R. P. Canale, Numerical methods for engineers, McGraw-Hill, New York, 1998.

[8] D. E. Goldberg, Genetic algorithms in search, optimization, and machine learning, Addison-Wesley, 1989.

[9] A. V. Oosterom, and J. Strackee, "The solid angle of a plan triangle," IEEE transactions on Biomedical Engineering, vol. BME-30, no 2, February 1983. 